\title{
COMPLETELY INJECTIVE SEMIGROUPS WITH CENTRAL IDEMPOTENTS
}

\author{
by E. H. FELLER and R. L. GANTOS $\dagger$ \\ (Received 23 October, 1967; revised 26 April, 1968)
}

1. Introduction. A right [left] unitary $S$-system is a set $M$ with right [left] operators in a semigroup $S$ with 1 , where $x 1=x[1 x=x]$ for all $x \in M$. We define a semigroup $S$ with 1 to be completely right [left] injective provided that every right [left] unitary $S$-system is injective. The main purpose of this paper is to determine a structure for completely right [left] injective semigroups whose idempotents are in the centre.

For a semigroup $S$ whose idempotents are in the centre, we prove that $S$ is completely right injective if and only if $S$ has a zero element and each right ideal is generated by an idempotent. These semigroups are exactly of the type that are unions of disjoint groups whose identity elements are dually well-ordered, and where the multiplication is determined by homomorphisms between groups. This is a special case of Theorem 4.11 of $[2, p .128]$.

2. Structure theorems. Since we are concerned with right unitary $S$-systems in this paper, we shall use the term $S$-system to mean " right unitary $S$-system". Consequently all semigroups in this paper will contain an identity element 1 . An $S$-system $M_{S}$ is injective if and only if, for every $S$-monomorphism $g: P_{S} \rightarrow R_{S}$ and for every $S$-homomorphism $h: P_{S} \rightarrow M_{S}$, there exists an $S$-homomorphism $h^{*}: R_{S} \rightarrow M_{S}$ such that $h^{*} g=h$. It follows that, if $N_{S}$ is an injective subsystem of $M_{S}$, then there exists an idempotent $S$-epimorphism $f: M_{S} \rightarrow N_{S}$.

2.1. Definition. A semigroup $S$ is called completely right [left] injective contains an identity element 1 and every right [left] unitary $S$-system is injective. $S$ is called completely injective if and only if it is both completely right and left injective.

2.2. THEOREM. If $S$ is completely right injective, then every right ideal of $S$ is generated by an idempotent.

Proof. If $H$ is a right ideal of $S$, then $H$ is an injective subsystem of $S_{S}$. Hence there exists an idempotent $S$-epimorphism $f: S \rightarrow H$. Thus $H=f(S)=f(1) S$ and from

$$
f(1) f(1)=f(1 \cdot f(1))=f(1)
$$

it follows that $f(1)$ is idempotent.

2.3. COROllary. Every completely right injective semigroup is regular.

Proof. This follows immediately from 2.2 and Lemma 1.13 of [2, p. 27].

$\dagger$ Research supported by the National Science Foundation under grant GP-6816 for the first author.

$\ddagger$ In [3], this term was used in the case of $S$-systems with zero. 


\subsection{COROLlaRY. Every completely right injective semigroup is semisimple.}

Proof. Let $A$ be an ideal of a completely right injective semigroup $S$. By 2.2, $A=e S$, where $e$ is idempotent. Then $A=e^{2} S \subseteq A^{2}$, and $A^{2}=A$. Hence, from exercise 7 of [2, p. 76], $S$ is semisimple.

It is known that the full transformation semigroup $T_{X}$ on a finite set $X$ of cardinal $n$ is a semisimple semigroup. However, for $n>2, T_{X}$ is not completely right injective, for not every right ideal is principal. Using the terminology of [1], an $S$-system $M$ is said to be weakly injective if and only if, for any right ideal $A$ of $S$ and $S$-homomorphism $f: A_{S} \rightarrow M_{S}$, there exists an element $z$ in $M$ such that $f(x)=z x$, for all $x$ in $A$.

2.5. Lemma. Let $S$ be a semigroup for which every right ideal of $S$ is generated by an idempotent. Then every $S$-system is weakly injective.

Proof. Let $M$ be an $S$-system, $A$ a right ideal of $S$, and $f: A_{S} \rightarrow M_{S}$ an $S$-homomorphism. By hypothesis, $A=e S$, for some idempotent $e \in S$. Setting $z=f(e)$, we have

for all $x$ in $A$.

$$
f(x)=f(e x)=f(e) x=z x,
$$

2.6. ThEOREM. Let $S$ be a semigroup whose idempotents are in the centre of $S$. Then $S$ is completely right injective if and only if $S$ has a zero and each right ideal of $S$ is generated by an idempotent.

Proof. Suppose that $S$ is completely right injective. By 2.2, every right ideal of $S$ is generated by an idempotent. Let $S^{0}$ denote the semigroup $S \cup 0$ defined as in $[3$, p. 4]. Clearly $S^{0}$ is an $S$-system containing $S$. By hypothesis, $S$ is an injective $S$-system and hence there exists an $S$-homomorphism $f: S^{0} \rightarrow S$ which extends the identity map, $1_{s}$, on $S$. For each $a \in S$, we have $f(0) a=f(0 a)=f(0)$. Thus $f(0)$ is a left zero element of $S$ and hence is idempotent. Since $f(0)$ belongs to the centre of $S$, it follows that $f(0)$ is the zero element of $S$ and $S=S^{0}$.

Conversely, assume that $S$ is a semigroup with zero whose idempotents belong to the centre of $S$ and that every right ideal of $S$ is generated by an idempotent. Let $M$ and $P \subseteq R$ be $S$-systems, and $f: P \rightarrow M$ be an $S$-homomorphism of $P$ into $M$. We show that $M$ is injective. Consider the set of all pairs $\left(P^{\prime}, f^{\prime}\right)$ consisting of subsystems $P^{\prime}$ of $R$ containing $P$ and $S$ homomorphisms $f^{\prime}$ of $P^{\prime}$ into $M$ which extend $f$. We partially order this set by the relation: $\left(P^{\prime}, f^{\prime}\right) \leqq\left(P^{\prime \prime}, f^{\prime \prime}\right)$ if and only if $P^{\prime} \subseteq P^{\prime \prime}$ and $f^{\prime \prime}$ extends $f^{\prime}$. Since any totally ordered subset has an upper bound in the set, the maximal principle applies to assure us of a maximal pair $\left(P_{0}, f_{0}\right)$. We prove that $P_{0}=R$.

Suppose that $P_{0} \subset R$ and let $r \in R$ be such that $r \notin P_{0}$. Set $A=\left\{a \in S \mid r a \in P_{0}\right\}$. We need to consider the two cases: $A$ non-empty or $A$ empty. For each case we will be able to define an $S$-homomorphism $h$ of $r S$ into $M$ which agrees with $f_{0}$ on $P_{0} \cap r S$.

Suppose that $A$ is non-empty; then $A$ is a right ideal of $S$ and, by hypothesis, $A=e S$, where $e$ is idempotent. Since $r \notin P_{0}$, it follows that $A \subset S$, and hence $e \neq 1$. The map $g: A \rightarrow M$ defined by $g(a)=f_{0}(r a) \quad(a \in A)$ is an $S$-homomorphism of $A$ into $M$. By $2.5, M$ is weakly injective. Thus, for some $z$ in $M$, we have $f_{0}(r a)=z a$ for all $a$ in $A$. 
Define $h: r S \rightarrow M$ by $h(r s)=z e s$ for all $s \in S$. We assert that $h$ is single-valued. Suppose that $r s_{1}=r s_{2}$. Since $e$ is central, $r e s_{1}=r s_{1} e=r s_{2} e=r e s_{2}$, and consequently

$$
h\left(r s_{1}\right)=z e s_{1}=f_{0}\left(r e s_{1}\right)=f_{0}\left(r e s_{2}\right)=z e s_{2}=h\left(r s_{2}\right) .
$$

Also $h=f_{0}$ on $P_{0} \cap r S$. Suppose that $x \in P_{0} \cap r S$. Then $x=r a \in P_{0}$, where $a \in A$. Since $e a=a$, we have $h(x)=h(r a)=z e a=z a=f_{0}(r a)=f_{0}(x)$. The map $h$ is clearly an $S$-homomorphism of $r S$ into $M$.

Suppose that $A$ is empty. Define $h: r S \rightarrow M$ by $h(x)=m 0$ for all $x \in r S$, where $m$ is an arbitrary but fixed element of $M$. Then $P_{0} \cap r S$ is empty and $h(x) s=(m 0) s=m 0=h(x s)$ for all $x \in r S$ and $s \in S$. Hence $h$ is also an $S$-homomorphism in this case.

Set $P^{*}=P_{0} \cup r S$, and let $f^{*}: P^{*} \rightarrow M$ be the map defined by $f^{*}(x)=f_{0}(x)$ for $x \in P_{0}$, and $f^{*}(x)=h(x)$ for $x \in r S$. By the above, $f^{*}$ is an $S$-homomorphism of $P^{*}$ into $M$ which extends $f_{0}$. Hence $\left(P^{*}, f^{*}\right)>\left(P_{0}, f_{0}\right)$, which contradicts the maximality of the pair $\left(P_{0}, f_{0}\right)$. Thus $P_{0}=R$, and $M$ is injective.

In the following two lemmas we assume that $S$ is a completely right injective semigroup such that every idempotent of $S$ is in the centre of $S$.

2.7. Lemma. The right ideals of $S$ are totally ordered and satisfy the ascending chain condition (A.C.C.). Moreover, the set of all idempotents $E$ of $S$, under the natural partial ordering, form a dually well-ordered set in the sense that every non-empty subset $K$ of $E$ contains a greatest element in $K$.

Proof. If $e S$ and $f S$ are two right ideals of $S$, then $e S \cup f S$ is a principal right ideal by 2.2 . Thus either $e S \cup f S=e S$ or $e S \cup f S=f S$, which implies that either $f S \subseteq e S$ or $e S \subseteq f S$.

If $\left\{e_{\alpha} S \mid \alpha \in I\right\}$ is a family of right ideals of $S$, then $\bigcup_{\alpha \in I} e_{\alpha} S=e_{\beta} S$ for some $\beta \in I$. Hence $S$ satisfies the A.C.C. for right ideals.

The natural partial ordering on $E$ is defined by $e \leqq f(e, f$ in $E)$ if and only if $e f=f e=e$. Since $E$ is commutative, $e \leqq f$ if and only if $e S \subseteq f S$. The well-ordering follows from the A.C.C.

2.8. Lemma. The semigroup $S$ is an inverse semigroup which is a union of disjoint groups.

Proof. By 2.3, $S$ is regular. Since the idempotents of $S$ commute, then, by Theorem 1.17 of [2, p. 28], $S$ is an inverse semigroup. Let $a \in S, e=a a^{-1}$ and $f=a^{-1} a$. Since $e$ and $f$ are central idempotents,

$$
e=e^{2}=a a^{-1} a a^{-1}=a f a^{-1}=f a a^{-1}=f e=\left(a^{-1} a\right) e=a^{-1} e a=a^{-1}\left(a a^{-1}\right) a=a^{-1} a=f
$$

Therefore $a=e a=a e$ and $a a^{-1}=a^{-1} a=e$ and these together imply that $a \in H_{e}$, the $\mathscr{H}$-class containing $e$. From Theorem 2.16 of $\left[2\right.$, p. 59], $H_{e}$ is a group. Thus $S$ is a union of groups.

We shall now establish the structure for our completely right injective semigroups, which is a special case of $[2$, p. 128]. We shall say that a set is dually well-ordered if and only if it is a semilattice in which every non-empty subset has a greatest element. 
2.9. StruCtURE THEOREM. Let $X$ be a dually well-ordered set containing a least element $o$ such that, for each $\alpha \in X$, there corresponds in a one-to-one manner a group $G_{\alpha}$ with identity $e_{\alpha}$, the group corresponding to o being the one-element group $\left\{e_{0}\right\}$. For each $\alpha, \beta$ of $X$ with $\alpha>\beta$, let there correspond a homomorphism $f_{\beta, \alpha}$ of $G_{\alpha}$ into $G_{\beta}$, such that, if $\alpha>\beta>\gamma$, then $f_{\gamma, \beta} f_{\beta, \alpha}=f_{\gamma, \alpha}$. Let $f_{\alpha, \alpha}$ be the identity mapping of $G_{\alpha}$ and let $S$ be the union of all the $G_{\alpha}(\alpha \in X)$. Define the product of $a_{\alpha}$ and $a_{\beta}$ in $S\left(a_{\alpha} \in G_{\alpha}, a_{\beta} \in G_{\beta}\right)$ by $a_{\alpha} a_{\beta}=f_{\gamma, \alpha}\left(a_{\alpha}\right) f_{\gamma, \beta}\left(a_{\beta}\right)$, where $\gamma=\alpha \beta=\alpha \wedge \beta$ in $X$.

Then $S$ is a completely right injective semigroup whose idempotents are in the centre of $S$. Conversely, every such semigroup is exactly of this form.

Proof. From Theorem 4.11 of [2, p. 128], $S$ is an inverse semigroup which is a union of groups. Thus the idempotents of $S$ are central. The element $e_{0}$ is the zero element of $S$ for $\alpha \geqq 0$, for all $\alpha \in X$, and $a_{\alpha} e_{0}=f_{\alpha 0, \alpha}\left(a_{\alpha}\right) f_{\alpha 0,0}\left(a_{0}\right)=e_{0} e_{0}=e_{0}$. From the product defined in $S$, the map $\alpha \rightarrow e_{\alpha}$ is an order preserving isomorphism of the semilattice $X$ onto the semilatice $E$ of idempotents of $S$, where $e \leqq f(e, f \in E)$ if and only if $e f=f e=e$. Consequently $\alpha \geqq \beta$ $(\alpha, \beta \in X)$ if and only if $e_{\alpha} \geqq e_{\beta}$. Since $X$ is dually well-ordered, $E$ is dually well-ordered.

Let $R$ be a right ideal of $S$. Since $S$ is regular, $R$ contains an idempotent. By the dual well-ordering of $E$, it follows that $R$ contains a greatest idempotent $e$. Thus $e S \subseteq R$. If $a \in R$, then $a a^{-1} \in R$. Hence $e a a^{-1}=a a^{-1}$, and $e a=a \in e S$. Therefore $e S=R$. It follows from 2.6 that $S$ is completely right injective.

The converse statement follows directly from $2.7,2.8$ and Lemmas 4.9 and 4.10 of [2, pp. 127-28].

2.10. PROPOSITION. If $S$ is a completely right injective semigroup whose idempotents are in the centre of $S$, then $S$ is completely left injective.

Proof. Let $L$ be a left ideal of $S$. If $a \in L S$, then $a=u s$, where $u \in L$ and $s \in S$. Since $S$ is an inverse semigroup and the idempotents of $S$ are central, it follows that

$$
a=u s=\left(u u^{-1} u\right) s=u s u^{-1} u=\left(u s u^{-1}\right) u \in L .
$$

Hence $L S \subseteq L$ and so $L$ is a two-sided ideal of $S$. Thus every left ideal of $S$ is generated by an idempotent. By the dual statement of 2.6 we have the proposition.

We now determine the explicit sets which form the maximal subgroups of 2.9 .

2.11. PROPOSITION. If $S$ is a completely right injective semigroup whose idempotents are in the centre of $S$, then the $\mathscr{H}$-classes of $S$, which are the maximal subgroups of $S$, are precisely the sets $e S \backslash f S$, where $e, f \in E$ and $f S$ is a maximal right ideal of $S$ contained in $e S$. The element $e$ is the identity of $e S \backslash f S$.

Proof. By 2.8, $S$ is an inverse semigroup which is a union of groups. This implies, as stated in [2, p. 127], that all the relations of Green are the same, and the equivalence classes are just the maximal subgroups of $S$. That is, if $a \in S$, then $H_{a}=R_{a}=L_{a}=D_{a}=J_{a}$, all being the maximal subgroup of $S$ containing $a$.

Let $f S$ be a maximal right ideal of $S$ contained in $e S$, where $e$ and $f$ are idempotents. Then $f<e$ and no idempotent of $S$ is between $f$ and $e$. If $a \in e S \mid f S$, then $a=e a$ and hence 
$a a^{-1}=e a a^{-1} \in e S . \quad$ Since $a a^{-1} \leqq e$ and the idempotents of $S$ are totally ordered, it follows that $a a^{-1}=e$. Hence $a \mathscr{R e}$ and so $e S \backslash f S \subseteq R_{e}$. On the other hand, if $b \in R_{e}$, then $b S=e S \supseteq f S$ and so $b \notin f S$. Therefore $e S \backslash f S=R_{e}=H_{e}$.

Conversely, let $H_{a}(a \in S)$ be an $\mathscr{H}$-class of $S$. Let $e \in H_{a}$ be the identity element of the group $H_{a}$. By 2.7, the right ideals of $S$ satisfy the A.C.C. Hence $e S$ contains a maximal right ideal $f S$ of $S$, where $f$ is idempotent. By the above, $e S \backslash f S$ is the $\mathscr{H}$-class containing $e$ and so $e S \backslash f S=H_{e}=H_{a}$.

For inverse semigroups, we have, from 2.2, 2.6, 2.8 and 4.8 of [2, p. 127],

2.12. Proposition. A semigroup $S$ with zero is an inverse semigroup which is a union of groups, and whose right ideals are principal if and only if $S$ is completely right injective with idempotents in the centre of $S$.

\section{REFERENCES}

1. P. Berthiaume, The injective hull of $S$-sets, Canad. Math. Bull. 10, no. 2 (1967), 261-273.

2. A. H. Clifford and G. B. Preston, The algebraic theory of semigroups, Amer. Math. Soc. Surveys, No. 7 (Providence, R.I., 1961). Nachr.

3. E. H. Feller and R. L. Gantos, Indecomposable and injective $S$-systems; to appear in $M a t h$.

\section{UNIVERSITY OF WISCONSIN}

MilWAUKEB 